\title{
A prospective comparison of UroVysion FISH and urine cytology in bladder cancer detection
}

\author{
Hugh J. Lavery', Boriana Zaharieva ${ }^{1,2}$, Andrew McFaddin $^{3}$, Nyla Heerema ${ }^{3}$ and Kamal S. Pohar ${ }^{1,2^{*}}$
}

\begin{abstract}
Background: UroVysion fluorescence in situ hybridization (UFISH) was reported to have superior sensitivity to urine cytology. However uFISH studies are limited by varying definitions of what is considered a positive result, absence of histopathology and small sample size. The aim of our study was to better determine the performance characteristics of uFISH and urine cytology by overcoming some of the deficiencies of the current literature.

Methods: Intraoperative bladder wash cytology and UFISH were collected prospectively on all patients. Strict definitions for positivity of UFISH and cytology were determined before initiating the study. A re-review of false-negative UFISH specimens was performed to analyze potential sources of error. Sixteen bladder tumors embedded in paraffin were analyzed by UFISH and compared with the result in the urine.

Results: One hundred and twenty-nine specimens were analyzed. Sensitivity was $67 \%$ and $69 \%(p=0.54)$; specificity was $72 \%$ and $76 \%$ ( $p=1.0$ ), for UFISH and cytology, respectively. Thirty-two false negative uFISH samples were re-reviewed. Low grade tumors often showed cells with abnormal morphology and patchy DAPI staining but diploid chromosomal counts and a few high grade tumors had tetraploid counts but less than needed to interpret uFISH as positive. UFISH study of the tumors revealed three categories; positive in both tumor and urine (9), negative in both tumor and urine (5) and positive in tumor but negative in urine (2).

Conclusion: In a pathologically-confirmed analysis of bladder washed urine specimens, UFISH does not outperform urine cytology in cancer detection.
\end{abstract}

Keywords: Urinary bladder neoplasm, In situ hybridization, Fluorescence, Cytology

\section{Background}

UroVysion fluorescence in situ hybridization (uFISH) is a multitarget, multicolor FISH assay that has been developed for the detection of urothelial carcinoma in the urine [1]. The assay is mostly used in the surveillance of patients with a history of bladder cancer [2-4]. Numerous studies have compared the performance characteristics (sensitivity and specificity) of uFISH to urinary cytology [5-12]. A recent meta-analysis of the published literature on uFISH reported superior sensitivity and comparable specificity when compared to cytology [13].

\footnotetext{
* Correspondence: kamal.pohar@osumc.edu

${ }^{1}$ Department of Urology, Ohio State University Wexner Medical Center, Columbus, $\mathrm{OH}$ 43210, USA

${ }^{2}$ Comprehensive Cancer Center, The Ohio State University, Columbus, $\mathrm{OH}$ 43210, USA

Full list of author information is available at the end of the article
}

uFISH is reported dichotomously (either positive or negative) based on criteria that include not only chromosomal changes but cell morphology. Despite the stringency of test reporting as either positive or negative, difficulty of interpretation of chromosomal and morphologic changes does exist, potentially influencing the usefulness of the test in cancer detection. Limitations of previous publications on uFISH include varying definitions of what is considered a positive result, lack of histopathology and small sample size [14-19]. Also the likelihood a given bladder cancer may not have the chromosomal alterations measured by uFISH and thus not capable of being detected by the test is not entirely known.

Similar to uFISH, urinary cytology is reported as positive or negative however additional terminology in reporting 
cytology specimens includes suspicious and atypical. The clinical significance of an atypical cytology has been debated and whether an atypical cytology is considered positive or negative influences the sensitivity of urine cytology in bladder cancer detection. A recent large scale study provides further evidence that classifying an atypical cytology as negative has little influence of the sensitivity of cancer detection [20].

The aim of our study was to better determine the performance characteristics of uFISH and urine cytology by overcoming some of the deficiencies of the current literature.

\section{Methods}

\section{Sample collection}

Institutional review board approval was obtained for an institutional tissue- and body fluid banking protocol. Patients were consented to allow for collection of urine specimens and fresh frozen and formalin-fixed paraffin embedded tumor tissue for research purposes, if not clinically needed, in a prospective manner on all patients undergoing transurethral resection of bladder tumor (TURBT) or radical cystectomy (RC). A total of 129 urine specimens were included in the study. Following induction of anesthesia and immediately prior to the surgical procedure, two $60 \mathrm{ml}$ bladder-washed aliquots of urine were obtained via a rigid cystoscope for TURBT or urethral catheter for RC. The two aliquots were randomly assigned for urine cytology or uFISH analysis.

In addition, 16 formalin fixed paraffin embedded (FFPE) bladder tumor specimens and 4 normal bladder urothelial samples were analyzed by uFISH. A representative area containing $\geq 80 \%$ tumor cells was selected for uFISH testing on each FFPE slide. All tumors evaluated in this study represented primary bladder cancers.

\section{uFISH and cytology}

All cytology specimens were analyzed by two ctyopathologists each with over 10 years of experience in urine cytology. Samples for conventional urine cytology were centrifuged and obtained by the Cytospin method. The slides were then stained by the standard Papanicolaou method. All uFISH specimens were processed according to the manufacturer's protocol by one cytogenetic technician and analyzed by one cytogeneticist with many years of experience in cytogenetics and cell morphology. The pathologists and cytogeneticist had no prior knowledge of this study. Tissue samples were evaluated by standard institutional protocol.

uFISH positivity in the bladder washings was determined by the criteria as listed in the package insert after all cells in a $15 \mathrm{~mm}$ diameter site were evaluated. Four or more abnormal cells that had chromosomal gain for at least two of chromosomes 3,7 or 17 or $\geq 12$ cells with homozygous loss of 9 p21 or samples with $\geq 10$ tetraploid cells with normal morphology were considered positive.

A previous unpublished institutional effort determined that urothelial cancer was proven histologically when the cytology was reported as positive in $9 / 9(100 \%)$, suspicious in 8/9 (89\%) and atypical in 1/13 (7\%) patients. Importantly all patients with a negative cytology all had a normal cystoscopy. Therefore for the purpose of this study a positive or suspicious cytology was considered positive and an atypical and negative cytology was considered negative. These definitions were generated prior to data analysis.

All TURBT were performed in a standardized manner that included an initial assessment of the entire bladder and prostatic urothelium and a pre- and post-resection exam under anesthesia. All areas of visible tumor as well as sites of previous resection were resected in their entirety. Random bladder and/or prostate urothelial biopsies were frequently performed. Upper urinary tract evaluation by urography was always performed and the presence of upper urinary tract carcinomas was excluded. Radical cystectomy was performed as previously reported [21]. Final histopathology from the operative specimen was considered the standard to which uFISH and cytology were compared.

At the completion of the study the cytogeneticist was unblinded and a post-hoc analysis of false-positive and false-negative uFISH was conducted. Information regarding cellularity, morphology, DAPI (4,6-Diamidino-2Phenylindole) staining, and aneuploidy of the uFISH chromosomes that was not presented in the initial report was collected. All false-negative uFISH slides were re-run with residual urine from the original aliquot to ensure quality control.

\section{uFISH in the tumors}

For FISH, 5- $\mu \mathrm{m}$ paraffin sections were placed in xylene for $30(3 \times 10)$ minutes followed by dehydration in $100 \%$ ethanol for $10(2 \times 5)$ minutes. Prior to hybridization, the slides were treated with Paraffin Pretreatment Reagent Kit (Abbott Molecular Inc., Des Plaines, IL, USA, Cat. \# 32-801,200) and dehydrated in 70\%, 85\%, and $100 \%$ alcohol solutions for $5 \mathrm{~min}$ each. FISH was performed using UroVysion probe (Abbott Molecular, Des Plaines, IL). Denaturation of the DNA was carried out at $75{ }^{\circ} \mathrm{C}$ for $10 \mathrm{~min}$ (probe mixture) or $5 \mathrm{~min}$ (slides). The probe mixture was applied to the slides and hybridized overnight in a moist chamber at $37{ }^{\circ} \mathrm{C}$. The post-hybridization washes were performed as described in 'LSI procedure' (Abbott Molecular Inc., Des Plaines, IL, USA). Slides were counterstained with $125 \mathrm{ng} / \mathrm{ml}$ DAPI in antifade. A minimum of 25 tumor cells were analyzed for copy number changes of chromosomes 3,7 , 17 and 9p21 [22]. If no abnormalities were detected, the 
remaining cells were counted until a sufficient number of cells with chromosomal abnormalities were found, or until 200 cells were evaluated, or the whole specimen was analysed. A positive result was the presence of $\geq 4$ cells with gains of two or more of chromosomes 3, 7 and 17 . In the case of chromosome 9 p21, a positive result was considered when $\geq 12$ cells showed absence of 9p21 signals.

\section{Statistics}

Data was collected and compiled using SPSS v.15.0. Statistical analyses were performed using McNemar's test for comparisons between uFISH and cytology and the chi-squared test for other comparisons. All $p$-values are two-sided.

\section{Results}

One hundred and twenty-nine urine samples were included in the study and collected at the time of TURBT $(n=94)$ or at RC $(n=35)$. Baseline characteristics of the study population and TURBT or RC pathology are listed in Table 1.

The indication for TURBT was suspicious cystoscopic findings for cancer in 48 (51\%) patients on surveillance for a history of non-muscle invasive bladder cancer (NMIBC), repeat TURBT within 6 weeks of the initial

Table 1 Patient and tumor characteristics

\begin{tabular}{ll}
\hline Mean Age (yrs) & 68 \\
Male:Female & $102: 27$ \\
TURBT & $94(73 \%)$ \\
Radical Cystectomy & $35(27 \%)$ \\
Indication for TURBT: & \\
Cystoscopic Abnormality: & $48(51 \%)$ \\
$\quad$ New diagnosis & 19 \\
$\quad$ Recurrence & 29 \\
Completion of initial TURBT & $38(40 \%)$ \\
Isolated positive cytology & $8(9 \%)$ \\
Tumor grade & \\
Low & $14(14 \%)$ \\
High & $87(86 \%)$ \\
Tumor stage & \\
T0 & $28(22 \%)$ \\
Ta & $31(24 \%)$ \\
CIS alone & $16(12 \%)$ \\
T1 & $19(15 \%)$ \\
T2 & $17(13 \%)$ \\
T3 & $15(12 \%)$ \\
Any CIS & $3(2 \%)$ \\
\hline
\end{tabular}

TURBT for confirming completeness of tumor resection in $38(40 \%)$ patients [23] and a positive urinary cytology with normal cystoscopic findings in 8 (9\%) patients. Eighty percent of the TURBTs were performed on patients who had previously been diagnosed with bladder cancer and $73(78 \%)$ specimens collected by TURBT identified the presence of cancer. All of the RC specimens had evidence for cancer except one specimen. A histologic diagnosis of cancer was present in 101 specimens (78\%).

Urine cytologies were reported as positive, consistent or suspicious for carcinoma or reported as negative, atypical or the presence of papillary clusters without fibrovascular cores. For the purpose of this study the definition of a positive or negative cytology is given in Table 2 along with the relative frequency of the cytologic descriptions. The interpretation of uFISH is described in the methods.

The overall performance of uFISH and cytology is shown in Table 3. uFISH was positive in 73 (56\%) collected specimens and cytology was positive in 76 (59\%). Overall sensitivity was $67 \%$ and $69 \%$ for uFISH and cytology $(p=0.54)$ and specificity was $72 \%$ and $76 \%$ for uFISH and cytology $(p=1.0)$, respectively. Sensitivity of the tests by tumor grade and stage is given in Table 4. Each of the sensitivity comparisons between uFISH and cytology was not statistically significant $(p>0.05)$. Similarly, no significant differences in sensitivity between uFISH and cytology were found when restricting the analysis to TURBT or RC procedures.

The grade and stage of the tumors of the 32 falsenegative uFISH specimens and 31 false negative cytologies are also presented in Table 4 . False negative results were noted in both low and high grade tumors as well as tumors of all pathologic stages, including carcinoma in situ. Specific analysis of the low grade Ta tumors identified a false negative result in 9 uFISH and 9 cytology specimens. Re-review of the uFISH slides from the false negative specimens often showed the presence of cells with abnormal morphology and patchy DAPI staining but diploid chromosomal counts and enough cellular material to interpret the test result as negative. In addition, of the 32 false-negative uFISH specimens, nine were from RC specimens. Each of the 9 RC specimens had significant papillary tumor burden on gross and microscopic

Table 2 Nomenclature for classification of urinary cytology reports Positive cytology $(n=76)$

Urothelial Carcinoma/Positive for malignancy (40)

Severely atypical cells consistent with high grade urothelial carcinoma (6) Suspicious for carcinoma (30)

Negative cytology $(n=53)$

No evidence of carcinoma/malignancy (32)

Urothelial atypia (18)

Papillary clusters without fibrovascular cores (3) 
Table 3 uFISH and cytology performance by stage

\begin{tabular}{llllll}
\hline Pathological stage & & Negative uFISH & Positive uFISH & Negative cytology & Positive cytology \\
\hline No Tumor & 28 & 21 & 7 & 22 & 6 \\
Tumor & $101^{\text {a }}$ & 32 & 66 & 31 & 70 \\
- Non-muscle invasive bladder cancer ( $\leq$ pT1) & $66^{\text {a }}$ & 23 & 40 & 22 & 44 \\
- Muscle invasive bladder cancer ( $\geq$ pT2) & 35 & 9 & 26 & 9 & 26 \\
\hline
\end{tabular}

aThree samples were not analyzed by uFISH due to lack of enough cellular material

pathology and final pathologic stage was pT2 (4), pT3 (4) and pT4 (1). Some of these same RC specimens also had the presence of carcinoma in situ (CIS). Re-review of the uFISH slides from these 9 specimens demonstrated three tumors with tetraploid counts but less than the number needed to interpret FISH as positive.

Analysis of the complementarity of the two tests was performed by examining the number of false-negatives from one test that were detected by the other. Fourteen (44\%) false-negative uFISH had positive cytologies, while $10(36 \%)$ false-negative cytologies had positive uFISH. Thus $64 \%$ of tumors missed by cytology would also have been missed by a concomitant uFISH. The sensitivity when either uFISH or cytology is considered positive was $82 \%$, while the specificity of such a definition was $59 \%$. If uFISH and cytology are both positive concurrently, the sensitivity decreases to $57 \%$ while specificity is $93 \%$.

Review of the longterm clinical history of the 7 falsepositive uFISH specimens revealed that three patients later underwent $\mathrm{RC}$ and another patient was recommended to undergo a RC for high risk of disease progression. Each of these four patients had muscle invasive disease with or without CIS when they recurred. Two additional patients developed upper urinary tract urothelial cancer and underwent a nephroureterectomy. The remaining patient has not recurred in more than two years of follow-up.

Four normal FFPE bladder urothelial samples and 16 FFPE tumor samples were evaluated by FISH to determine how often the chromosomal changes quantitated by the UroVysion FISH assay were present in the tumor. About $20 \%$ of the low and high grade tumors and $20 \%$ of the NMIBC and MIBC included in the study were chosen to measure UroVysion FISH positivity. All four normal samples analyzed were uFISH negative because of normal copy number counts. The uFISH data for both the tumor and the corresponding urine specimen are presented in Table 5 . Importantly 5 of 16 tumors (31\%) were uFISH negative and the corresponding urine sample for these tumors was also uFISH negative. The uFISH negative tumors included tumors of low and high grade as well as superficial and invasive disease. Also two tumors determined to be uFISH positive had a corresponding uFISH negative urine sample, including one high grade tumor.

\section{Discussion}

The main reported advantage of uFISH is increased sensitivity of cancer detection when compared to urine cytology, especially in high grade tumors [18, 24]. However, false-negative results still occur and may be due to failure of tumor detection at cystoscopy or an "anticipatory positive" UroVysion FISH [25]. In addition, in order for uFISH to identify the presence of a cancer it is necessary the cancer has gains of at least two of chromosomes 3, 7 or 17 in at least four or loss of 9p21 in at least twelve, morphologically abnormal cells, in accordance with the FDA approval of the uFISH assay $[1,5]$. And in fact, our study suggests not all bladder cancers have the chromosomal alterations detected by uFISH.

In addition, the uFISH assay is also associated with false positive results. One explanation for false positive results is the presence of tetraploidy in the uFISH assay that may reflect normal cell division or a proliferative response of the bladder epithelium to an inflammatory insult and not a cancerous process. Zellweger et al. determined the number of uFISH false positives can be reduced by establishing the presence of tetraploidy in at least 10

Table 4 Sensitivity of UFISH and cytology by grade and stage

\begin{tabular}{lllllll}
\hline & $\mathrm{n}^{\text {a }}$ & uFISH sensitivity (\%) & Cytology sensitivity (\%) & $p$-value & False negative uFISH $(n=32)$ & False negative cytology $(n=31)$ \\
\hline Low Grade & 14 & $25 \%$ & $36 \%$ & 0.63 & 9 & 9 \\
High Grade & 87 & $73 \%$ & $75 \%$ & 0.82 & 23 & 22 \\
Any CIS & 47 & $79 \%$ & $83 \%$ & 0.73 & 10 & 8 \\
CIS alone & 16 & $75 \%$ & $81 \%$ & 1.0 & 4 & 3 \\
SpT1 & $66^{\text {a }}$ & $63 \%$ & $67 \%$ & 0.45 & 23 & 22 \\
$\geq$ pT2 & 35 & $74 \%$ & $74 \%$ & 1.0 & 9 & 9 \\
\hline
\end{tabular}

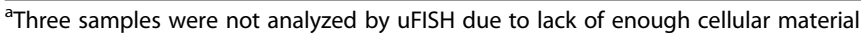

*Comparison of UFISH and Cytology sensitivity 
Table 5 uFISH results in formalin-fixed paraffin embedded (FFPE) tumors and corresponding urine samples

\begin{tabular}{llllll}
\hline Tumors & & $\begin{array}{l}\text { UFISH negative in } \\
\text { FFPE tumors }\end{array}$ & $\begin{array}{l}\text { UFISH negative in } \\
\text { urine samples }\end{array}$ & $\begin{array}{l}\text { UFISH positive in } \\
\text { FFPE tumors }\end{array}$ & $\begin{array}{l}\text { UFISH positive in } \\
\text { urine samples }\end{array}$ \\
\hline Low Grade & 2 & 1 & 2 & 1 & 0 \\
High Grade & 14 & 4 & 5 & 10 & 9 \\
$\leq$ pT1 & 9 & 1 & 2 & 8 & 7 \\
$\geq$ pT2 & 7 & 4 & 5 & 3 & 2 \\
\hline
\end{tabular}

morphologically abnormal cells as this is more reflective of the presence of cancer [26]. The importance of defining the number of cells with tetraploidy in the uFISH assay was further supported by the work of Kipp et al. [27]. Patients in both studies with a normal cystoscopy and a positive uFISH result based on defining teteraploidy in at least 10 cells were more likely to be diagnosed with cancer at the time of the original bladder biopsy or subsequent bladder biopsy thus improving upon both the sensitivity and specificity of the uFISH assay. Based on these publications our institution and others include tetraploidy in at least 10 morphologically abnormal cells in the definition of a positive uFISH result, in addition to the FDA approved criteria [27].

Our discussion mostly focuses on the false negative result and sensitivity of the uFISH assay. An extensive review by Jones of nine uFISH studies reported a sensitivity of $96 \%$ in CIS lesions (44 specimens) and $94 \%$ in 87 invasive tumor specimens [28]. Promotional literature for the UFISH test kit reports a "near 100\% sensitivity for dangerous high grade lesions" [17]. However our study findings are not consistent with these reports as we found a sensitivity of $79 \%$ in 47 CIS lesions and $74 \%$ in 35 invasive lesions. Moreover, our study findings are similar to other studies that indicate the performance of traditional urine cytology is similar to uFISH in bladder cancer detection $[29,30]$.

Why do our results of uFISH sensitivity differ from some other reports? Our study attempted to reduce the limitations of previous publications that may have included varying definitions of what is considered a positive uFISH result, lack of histopathology, heterogeneous patient population and small sample size. In our study all patients were evaluated in the operating room for presumed bladder cancer and had concurrent bladder washed urine samples thus increasing the cellularity of the specimen and histopathology for comparison. Only $14 \%$ of patients had low-grade tumors and $27 \%$ of specimens were acquired at the time of radical cystectomy for invasive disease. Therefore the patients included in the study presumably have a high likelihood of a positive $\mathrm{uFISH}$ and are more representative of the true performance characteristics of uFISH.

After the study concluded we validated our uFISH sensitivity analysis by re-evaluating the false negative cases. To this end a single experienced cytogenetic technician repeated the analysis of all false-negative specimens from stored residual urine and compared the result to the originally processed urine sample for the same patient. All false negative uFISH urine samples were confirmed to be negative on repeat evaluation. Importantly several samples contained morphologically abnormal cells and tetraploidy in less than 10 cells of the targeted probes and absence of the FDA approved criteria for a positive test result. We also recognized the urine samples obtained from most low grade tumors were comprised of morphologically abnormal cells with patchy DAPI nuclear staining but had normal diploid counts of the Urovysion markers. Therefore it is possible that bladder tumors with a negative urine uFISH do not have the chromosomal alterations that the uFISH test is detecting.

To investigate this possibility we directly performed uFISH analysis of 16 bladder tumors of varying grades and stages. Our findings determined that about 30\% of bladder tumors do not have aneuploidy of chromosomes $3,7,17$ or 9 p21 and may explain some of the uFISH false negatives in our evaluation of the urine samples in not only high grade tumors but also low grade tumors. However, our study determined that all tumors, with two exceptions, with the uFISH chromosomal alterations can be detected in the corresponding urine sample. The findings of our study suggest the need for a more comprehensive cytogenetic evaluation of bladder cancer is needed to improve FISH based testing. The benefit of using complementary molecular tests in the urine such as loss of heterozygosity, nuclear matrix protein 22 (NMP22) and array comparative genomic hybridization (array CGH) has been shown in the literature [31-33]. Therefore, as the cost of next-generation sequencing, microarray-based $\mathrm{CGH}$ and other global approaches decreases and becomes more clinically applicable these technologies should be studied alone or as a complement to uFISH to improve bladder cancer detection.

As a final comment, in our study the sensitivity of urine cytology was much higher in high grade tumors when compared to low grade tumors. This was an expected result however we did note a higher sensitivity (36\%) for urine cytology in low grade tumors in our study when compared to prior publications. The likely explanation of 
a positive cytology in the six patients with low grade tumors in this study is the fact that five of the patients were defined as intermediate risk tumors characterized by multifocality and tumors were greater than $3 \mathrm{~cm}$ in size. The collection of the urine samples by washing or barbotage is known to increase cellular yield of the sample. Therefore when the burden of tumor is high, even in the context of low grade tumors, it is more likely a positive cytology will be obtained when the specimen is collected by barbotage compared to a voided sample.

Our study design is clearly limited by its lack of clinical applicability. However, the study was purposely designed to advance the current state of knowledge of uFISH by determining its true performance characteristics as it is used today and to recognize opportunities for improvement. The study provides valuable new insights on improving FISH based assays and the need to better standardize definitions of reporting a positive result.

\section{Conclusions}

In a prospective, pathologically-confirmed analysis of intraoperative bladder washed urine specimens, Urovysion FISH does not outperform traditional urine cytology in either sensitivity or specificity.

\section{Abbreviations \\ CGH: Comparative genomic hybridization; CIS: Carcinoma in situ; DAPI: 4,6- Diamidino-2-Phenylindole; LSI: Locus Specific Identifier; NMP22: nuclear matrix protein 22; OR: Operating room; RC: Radical cystectomy; TURBT: Transurethral resection of bladder tumor; $\mathrm{UFISH}$ : UroVysion fluorescence in situ hybridization}

Acknowledgements

Not applicable.

Funding

This study did not receive any extra-institutional funding.

\section{Availability of data and materials}

The datasets supporting the conclusions of this article are included within the article. Any request of data and material may be sent to the corresponding author.

\section{Authors' contributions}

The study was designed by KSP and HJL. The collection of data was performed by KSP, HJL, AM and BZ. The UroVysion FISH results were obtained and interpreted by $\mathrm{AM}, \mathrm{BZ}$ and $\mathrm{NH}$. The data analysis was performed by KSP, $\mathrm{HJL}, \mathrm{AM}, \mathrm{BZ}$ and $\mathrm{NH}$. The manuscript was written and critically revised by KSP, $\mathrm{HJL}, \mathrm{BZ}, \mathrm{AM}$ and $\mathrm{NH}$. All authors read and approved the final manuscript.

\section{Competing interests}

The authors declare that they have no competing interests.

\section{Consent for publication}

Not applicable.

\section{Ethics approval and consent to participate}

Written informed consent was obtained from all patients before treatment according to our institutional guidelines. The study was approved by The Ohio State University institutional review board.

\section{Publisher's Note}

Springer Nature remains neutral with regard to jurisdictional claims in published maps and institutional affiliations.

\section{Author details}

'Department of Urology, Ohio State University Wexner Medical Center, Columbus, OH 43210, USA. ${ }^{2}$ Comprehensive Cancer Center, The Ohio State University, Columbus, $\mathrm{OH} 43210$, USA. ${ }^{3}$ Department of Pathology, Division of Cytogenetics, Ohio State University Wexner Medical Center, Columbus, $\mathrm{OH}$ 43210, USA.

Received: 21 November 2016 Accepted: 23 March 2017

Published online: 07 April 2017

\section{References}

1. Sokolova IA, Halling KC, Jenkins RB, Burkhardt HM, Meyer RG, Seeling SA, et al. The development of a multitarget, multicolor fluorescence in situ hybridization assay for the detection of urothelial carcinom in urine. J Mol Diagn. 2000:2:116-23.

2. Gofrit ON, Zorn KC, Silvestre J, Shalhav AL, Zagaja GP, Msezane LP, et al. The predictive value of multi-targeted fluorescent in-situ hybridization in patients with history of bladder cancer. Urol Oncol. 2008;26:246-9.

3. Maffezzini M, Campodonico F, Capponi G, Canepa G, Casazza S, Bandelloni $\mathrm{R}$, et al. Prognostic significance of fluorescent in situ hybridisation in the follow-up of non-muscle-invasive bladder cancer. Anticancer Res. 2010;30:4761-5.

4. Lotan Y, Shariat SF, Schmitz-Dräger BJ, Sanchez-Carbayo M, Jankevicius F, Racioppi $\mathrm{M}$, et al. Considerations on implementing diagnostic markers into clinical decision making in bladder cancer. Urol Oncol. 2010;28:441-8.

5. Halling KC, King W, Sokolova IA, Meyer RG, Burkhardt HM, Halling AC, et al. A comparison of cytology and fluorescence in situ hybridization for the detection of urothelial carcinoma. J Urol. 2000;164:1768-75.

6. Sarosdy MF, Schellhammer P, Bokinsky G, Kahn P, Chao R, Yore L, et al. Clinical evaluation of a multi-target fluorescent in situ hybridization assay for the detection of bladder cancer. J Urol. 2002:168:1950-4.

7. Skacel M, Fahmy M, Brainard JA, Pettay JD, Biscotti CV, Liou LS, et al. Multitarget fluorescence in situ hybridization assay detects transitional cell carcinoma in the majority of patients with bladder cancer and atypical or negative urine cytology. J Urol. 2003;169:2101-5.

8. Sarosdy MF, Kahn PR, Ziffer MD, Love WR, Barkin J, Abara EO, et al. Use of a multitarget fluorescence in situ hybridization assay to diagnose bladder cancer in patients with hematuria. J Urol. 2006;176:44-7.

9. Bergman J, Reznichek RC, Rajfer J. Surveillance of patients with bladder carcinoma using fluorescent in-situ hybridization on bladder washings. BJU Int. 2008;101:26-9.

10. Fritsche HM, Burger M, Dietmaier W, Denzinger S, Bach E, Otto W, et al. Multicolor FISH (UroVysion) facilitates follow-up of patients with high-grade urothelial carcinoma of the bladder. Am J Clin Pathol. 2010;134:597-603.

11. Caraway NP, Khanna A, Fernandez RL, Payne L, Bassett Jr RL, Zhang HZ, et al. Fluorescence in situ hybridization for detecting urothelial carcinoma: a clinicopathologic study. Cancer Cytopathol. 2010;118:259-68.

12. Galván AB, Salido M, Espinet B, Placer J, Pijuan L, Juanpere N, et al. A multicolor fluorescence in situ hybridization assay: a monitoring tool in the surveillance of patients with a history of non-muscle-invasive urothelial cell carcinoma: a prospective study. Cancer Cytopathol. 2011;119:395-403.

13. Hajdinjak T. UroVysion FISH test for detecting urothelial cancers: meta-analysis of diagnostic accuracy and comparison with urinary cytology testing. Urol Oncol. 2008;26:646-51.

14. Veeramachaneni $R$, Nordberg ML, Shi R, Herrera GA, Turbat-Herrera EA. Evaluation of fluorescence in situ hybridization as an ancillary tool to urine cytology in diagnosing urothelial carcinoma. Diagn Cytopathol. 2003;28:301-7.

15. Varella-Garcia M, Akduman B, Sunpaweravong P, Di Maria MV, Crawford ED. The UroVysion fluorescence in situ hybridization assay is an effective tool for monitoring recurrence of bladder cancer. Urol Oncol. 2004;22:16-9.

16. Daniely M, Rona R, Kaplan T, Olsfanger S, Elboim L, Zilberstien Y, et al. Combined analysis of morphology and fluorescence in situ hybridization significantly increases accuracy of bladder cancer detection in voided urine samples. Urology. 2005;66:1354-9.

17. Krause FS, Rauch A, Schrott KM, Engehausen DG. Clinical decisions for treatment of different staged bladder cancer based on multitarget fluorescence in situ hybridization assays? World J Urol. 2006:24:418-22.

18. Bubendorf L, Grilli B, Sauter G, Mihatsch MJ, Gasser TC, Dalquen P. Multiprobe FISH for enhanced detection of bladder cancer in voided urine specimens and bladder washings. Am J Clin Pathol. 2011;116:79-86. 
19. Dimashkieh H, Wolff DJ, Smith TM, Houser PM, Nietert PJ, Yang J. Evaluation of urovysion and cytology for bladder cancer detection: a study of 1835 paired urine samples with clinical and histologic correlation. Cancer Cytopathol. 2013;121:591-7.

20. Brimo F, Vollmer RT, Case B, Aprikian A, Kassouf W, Auger M. Accuracy of urine cytology and the significance of an atypical category. Am J Clin Pathol. 2009;132:785-93.

21. Dangle PP, Gong MC, Bahnson RR, Pohar KS. How do commonly performed lymphadenectomy templates influence bladder cancer nodal stage? J Urol. 2010;183:499-503.

22. UroVysion fluorescence in situ hybridization promotional literature and package insert, Abbott molecular. Des Plaines. IL, USA.

23. NCCN bladder cancer guidelines (2017). Available from https://www.nccn. org/professionals/physician_gls/f_guidelines.asp.

24. Reid-Nicholson MD, Ramalingam P, Adeagbo B, Cheng N, Peiper SC, Terris MK. The use of Urovysion fluorescence in situ hybridization in the diagnosis and surveillance of non-urothelial carcinoma of the bladder. Mod Pathol. 2009;22:119-27.

25. Yoder BJ, Skacel M, Hedgepeth R, Babineau D, Ulchaker JC, Liou LS, et al. Reflex UroVysion testing of bladder cancer surveillance patients with equivocal or negative urine cytology: a prospective study with focus on the natural history of anticipatory positive findings. Am J Clin Pathol. 2007;127:295-301.

26. Zellweger T, Benz G, Cathomas G, Mihatsch MJ, Sulser T, Gasser TC, et al. Multi-target fluorescence in situ hybridization in bladder washings for prediction of recurrent bladder cancer. Int J Cancer. 2006;119:1660-5.

27. Kipp BR, Halling KC, Campion MB, Wendel AJ, Karnes RJ, Zhang J, et al. Assessing the value of reflex fluorescence in situ hybridization testing in the diagnosis of bladder cancer when routine urine cytological examination is equivocal. J Urol. 2008;179:1296-301.

28. Jones JS. DNA-based molecular cytology for bladder cancer surveillance. Urology. 2006;67:35-45.

29. Kamat AM, Karam JA, Grossman HB, Kader AK, Munsell M, Dinney CP. Prospective trial to identify optimal bladder cancer surveillance protocol: reducing costs while maximizing sensitivity. BJU Int. 2011;108:1119-23.

30. Youssef RF, Schlomer BJ, Ho R, Sagalowsky Al, Ashfaq R, Lotan Y. Role of fluorescence in situ hybridization in bladder cancer surveillance of patients with negative cytology. Urol Oncol. 2012;30:273-7.

31. Wild PJ, Fuchs T, Stoehr R, Zimmermann D, Frigerio S, Padberg B, et al. Detection of urothelial bladder cancer cells in voided urine can be improved by a combination of cytology and standardized microsatellite analysis. Cancer Epidemiol Biomark Prev. 2009;18:1798-806.

32. Kehinde EO, Al-Mulla F, Kapila K, Anim JT. Comparison of the sensitivity and specificity of urine cytology, urinary nuclear matrix protein-22 and multitarget fluorescence in situ hybridization assay in the detection of bladder cancer. Scand J Urol Nephrol. 2011;45:113-21.

33. Panzeri E, Conconi D, Antolini L, Redaelli S, Valsecchi MG, Bovo G, et al. Chromosomal aberrations in bladder cancer: fresh versus formalin fixed paraffin embedded tissue and targeted FISH versus wide microarray-based CGH analysis. PLoS One. 2011;6:e24237.

\section{Submit your next manuscript to BioMed Central and we will help you at every step:}

- We accept pre-submission inquiries

- Our selector tool helps you to find the most relevant journal

- We provide round the clock customer support

- Convenient online submission

- Thorough peer review

- Inclusion in PubMed and all major indexing services

- Maximum visibility for your research

Submit your manuscript at www.biomedcentral.com/submit 